Cite this: RSC Advances, 2013, 3, 1468

\title{
A comparative study of two amphiphilic merocyanines: from monomers to aggregates in Langmuir and Langmuir-Blodgett mixed films $\dagger$
}

\author{
Andrea Lodi, ${ }^{a}$ Fabio Momicchioli, ${ }^{a}$ Monica Caselli, ${ }^{a}$ Gabriele Giancane ${ }^{b}$ \\ and Glauco Ponterini*a
}

\begin{abstract}
The Langmuir-Blodgett (LB) films with J aggregates of the amphiphilic MC2 merocyanine have been deeply investigated for more than two decades, mainly because of their interest for applications in photonic devices. To extend the excitation/emission wavelength range, we have studied an homologous dye, MC1, based on a shorter merocyanine chromophore, aiming at checking the ability of this compound to arrange into J aggregates within stable LB films. In this comparative MC1/MC2 investigation, we have addressed the structural and spectroscopic properties of the monomers in solution, the thermodynamic and morphological properties of the Langmuir monolayers at the air-water interface and the spectroscopic and photophysical properties, and the structural features thence obtainable, of the aggregates in the LB films. ${ }^{1} \mathrm{H}$ NMR experiments have shown that, in chloroform solutions, both dyes adopt planar conformations with the hydrophilic and hydrophobic groups pointing to opposite directions. Strongly attractive interactions are exhibited by both dyes towards arachidic acid in monolayers at the airsubphase interface, with a maximum stability for the $1: 2 \mathrm{MC}(1 / 2)$ : AA mixture. Relatively homogeneous LB multilayers have been obtained from $1: 4$ and $1: 2 \mathrm{MC}:$ AA mixtures with good transfer ratios. Absorption and emission of the LB films of MC1 are dominated by J aggregates, while those of MC2 show some $\mathrm{H}$ aggregate contributions too. The $\mathrm{J}$ aggregates of both compounds exhibit a tendency to align with the transition dipoles along the film dipping direction, only slightly tilted relative to the substrate surface; however, this tendency is more pronounced for the MC1 J aggregates. Overall, MC1 yields slightly more stable monolayers and more solid multilayers compared to MC2.
\end{abstract}

Received 20th November 2012, Accepted 20th November 2012

DOI: $10.1039 / \mathrm{c} 2 \mathrm{ra} 22962 \mathrm{e}$

www.rsc.org/advances approach builds-up mixed monolayers of merocyanine and fatty acids (and/or other appropriate components) on a pure water subphase. ${ }^{11,18}$ Several studies combining the two approaches have been also reported..$^{10,14,15}$

Because of the crucial role of the optical properties of merocyanines and their aggregates in the applications of their LB films, it is important to extend the wavelength range for these applications beyond that of $\mathrm{MC} 2$, without compromising LB film stability. To this aim, we have obtained a shorter and more rigid quasi-homologue of merocyanine, MC1 in Fig. 1, which absorbs and emits, as a monomer, around $100 \mathrm{~nm}$ to the blue of MC2 in a chloroform solution. Both dyes exhibited the formation of $\mathrm{H}$-type aggregates in films cast from such solutions. ${ }^{2}$ Also, their solubilization in water by complexation with $p$-sulfonated calixarenes has been investigated in view of the preparation of electrostatically self-assembled multilayers incorporating these dyes. ${ }^{19}$

In this contribution, we switch back to LB films of MC1 and MC2 following a combined version of the two approaches outlined above to promote dye aggregation.
${ }^{a}$ Dipartimento di Chimica, Università di Modena and Reggio Emilia and
Campi 183, 41100 Modena Italy. E-mail: glauco.ponterini@unimore.it

${ }^{b}$ Dipartimento di Beni Culturali, Università del Salento, 73100 Lecce Italy

† Electronic Supplementary Information (ESI) available. See DOI: 10.1039/ c2ra22962e 

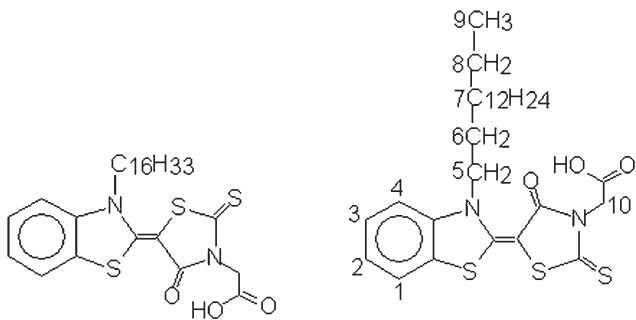

$\mathrm{MC} 1$
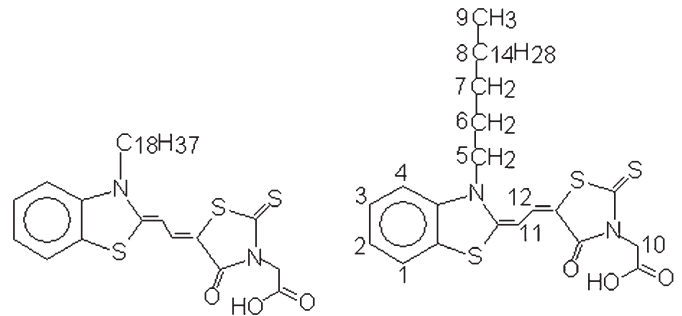

$\mathrm{MC} 2$

Fig. 1 Schematic representations of the conformers of MC1 obtained by torsion around the central double bond and of the two conformers of MC2 $(\pi, \pi, \pi$ on the left and $0, \pi, 0$ on the right, according to the notation in ref. 12) that are consistent with the ${ }^{1} \mathrm{H}$ NMR results (Section 3.1).

After an ${ }^{1} \mathrm{H}$ NMR characterization of the structures, and a comparative study of absorption and fluorescence properties of the two dyes as monomers in solution, we describe the thermodynamics of merocyanine-arachidic acid mixing in Langmuir monolayers formed on a subphase containing ammonia, and some spectroscopically monitored structural features of the LB films, including the extent of dye aggregation within them. The effects of the differences between the two merocyanines on these properties are highlighted and discussed.

\section{Experimental section}

MC1 was a gift from Dr I. Delprato, of Imation s.p.a., Ferrania (Italy). MC2 was purchased from Hayashibara Biochemical Laboratories, Inc., Okayama, Japan. Arachidic acid (AA, $\geqslant 99 \%$ ) was obtained from Aldrich. Chloroform, acetonitrile, methanol and cyclohexane (Fluka, Merck, Lab Scan) were all of spectroscopic grade. All compounds and solvents were used as received.

${ }^{1} \mathrm{H}$ NMR spectra were measured on a Bruker Avance400 spectrometer equipped with a $5 \mathrm{~mm}$ broad-band inverse probe head and operating at 9.4 $\mathrm{T}$. The spectra were acquired by a standard pulse sequence. Proton-proton transient nuclearOverhauser-effect (NOE) spectra were acquired using a twodimensional standard NOESY sequence with a mixing time of $0.8 \mathrm{~s}$.

The surface pressure/area per molecule isotherms were measured on a $612 \mathrm{D}$ Nima instrument at $20{ }^{\circ} \mathrm{C}$. The subphase was a $0.01 \%$ ammonia solution in ultra-pure water (Milli-Q system, resistivity larger than $18 \mathrm{M} \Omega \mathrm{cm}), \mathrm{pH}=10.3 .^{20}$ Chloroform solutions $\left(5 \times 10^{-4} \mathrm{M}\right)$ of MC1 or MC2 were sprayed on the aqueous subphase either pure or mixed with an AA chloroform solution in different $\mathrm{MC}$ : AA mole ratios: $4: 1$, $2: 1,1: 1,1: 2,1: 4$. The rate of compression of the floating monolayer was $20 \mathrm{~cm}^{2} \mathrm{~min}^{-1}$. Brewster Angle Microscopy (BAM) was carried out using a NFT BAM2plus system with a lateral resolution of $2 \mu \mathrm{m}$. Quartz plates for film deposition were made hydrophilic by immersion in a sulfochromic mixture for $15 \mathrm{~min}$, followed by sonication in a ChemSolv mixture (hydroalcoholic $\mathrm{KOH}$ solution ${ }^{21}$ ) for 60 min. Y-type LB depositions were made at $20{ }^{\circ} \mathrm{C}$ with immersion and emersion rates of 1 and $3 \mathrm{~mm} \mathrm{~min}^{-1}$, respectively.

UV-visible absorption spectra were measured on a Cary 100 Varian or a $\lambda 15$ Perkin Elmer spectrophotometer, equipped with film polarizers for linear dichroism measurements. Fluorescence measurements were performed on a Spex Jobyn-Yvon FloroMax2 spectrofluorometer. Experiments were carried out in $10 \times 10 \mathrm{~mm}^{2}$ cuvettes. Fluorescence measurements of the films were performed with a $50-60^{\circ}$ incidence angle of the excitation beam to maximize the emitted/scattered light ratio. The fluorescence quantum yields $\left(\Phi_{\mathrm{F}}\right)$ were determined at $24{ }^{\circ} \mathrm{C}$ relative to acridine orange hydrochloride in ethanol $\left(\Phi_{\mathrm{F}}=0.46\right)^{22}(\mathrm{MC} 1)$ and to eosin in ethanol $\left(\Phi_{\mathrm{F}}=\right.$ $0.72)^{23}$ (MC2).

The morphology of the film surfaces was explored using atomic force microscopy (AFM) in tapping mode (CP Park Autoprobe) in air and at room temperature. Typically, tips had a $3 \times 10^{-2} \mathrm{~N} \mathrm{~m}^{-1}$ force constant and a resonance frequency of $10 \times 10^{3} \mathrm{~Hz} ; 1-2$ scans, each one including 256 data points, were made per second. The surface roughness was considered equal to the standard deviation of the $z$-value distribution within a suitably large image area and was obtained through the PSI ProScan Image Processing software.

\section{Results and discussion}

\subsection{Structures and absorption/emission spectra of the monomers in solution}

By a detailed comparative analysis of X-ray crystallographic studies and both observed and calculated (DFT-B3LYP) IR solution spectra, Ikegami and $\operatorname{Kuroda}^{12}$ arrived at the conclusion that the dominant conformation of MC2 is most likely the $(\pi, \pi, \pi)$ one, i.e., the left one in Fig. 1. We have performed ${ }^{1} \mathrm{H}$ NMR of MC2 in deuterated chloroform to test this structural conclusion. The chemical shifts and spin-spin scalar coupling constants, $\delta$ and $J$, are reported in Table 1 (the atom numbering is in Fig. 1). A single signal pattern was observed, which is an indication of a dominant conformer in

Table $1{ }^{1} \mathrm{H}$ NMR chemical shifts $(\delta$, ppm) and spin-spin coupling constants (J, $\mathrm{s}^{-1}$ ) for merocyanine $\mathrm{MC2}$ in $\mathrm{CDCl}_{3}$. See Fig. 1 for the atom numbering

\begin{tabular}{ccccccccccc}
$\delta_{1}$ & $\delta_{2}$ & $\delta_{3}$ & $\delta_{4}$ & $\delta_{5}$ & $\delta_{6}$ & $\delta_{7}$ & $\delta_{8}$ & $\delta_{9}$ & $\delta_{10}$ & $\delta_{11}$ and $\delta_{12}$ \\
\hline 7.55 & 7.23 & 7.41 & 7.12 & 3.99 & 1.83 & 1.45 & $1.25-1.45$ & 0.92 & 4.97 & $5.39,7.72$ \\
$J_{1,2}$ & $J_{2,3}$ & $J_{3,4}$ & & $J_{5,6}$ & & & & & $J_{11,12}$ \\
7.7 & $\sim 7.9$ & 8.1 & & $\sim 7.8$ & & & & & &
\end{tabular}


solution. Assignment of all signals, apart from the two doublets at 5.39 and $7.72 \mathrm{ppm}$, results unambiguously from the chemical shifts, the multiplicities and the $J$ coupling constants. To confirm the assignments, NOE was observed between signals 1 and 2, 2 and 3, 3 and 4, 4 and 5, 4 and 6, 5 and 6. The two doublets at 5.39 and $7.72 \mathrm{ppm}$ of MC2 are assignable to the central methine protons. The scalar coupling constant between them is $12.7 \mathrm{~Hz}$, a value that points to an strans conformation. ${ }^{24}$ There are four conformations consistent with this indication, ${ }^{12}$ but they can be reduced to the two shown in Fig. 1. In fact, the most deshielded doublet is likely attributable to a proton close to the carbonyl function of the five-membered cycle of MC2. ${ }^{25}$ Also, NOE was clearly observed between both methylene protons 5 and 6 and the doublet at $5.39 \mathrm{ppm}$. These observations restrict the possible conformations for MC2 in solution to the two shown in Fig. 1: the doublet at $5.39 \mathrm{ppm}$ would be assigned to the proton bound to $\mathrm{C}_{11}$ for the conformation on the left and to that bound to $\mathrm{C}_{12}$ for the other conformation.

A preference for the conformation on the left $(\pi, \pi, \pi$ according to the notation in ref. 12) is suggested by the following considerations. Funakoshi et al. have investigated the ${ }^{1} \mathrm{H}$ NMR spectra of several merocyanines similar to MC2. ${ }^{25}$ For three compounds, they were able to observe two different sets of signals that they attributed to two conformations corresponding to those in Fig. 1 for MC2. The differences between the central methine proton signals were lower than 1 ppm for conformations corresponding to the right one, and were larger than $1.5 \mathrm{ppm}$ for the other conformation. The 2.33 ppm difference found for MC2 therefore points to the left-side conformation in Fig. 1 being the one found in solution. This assignment is in keeping with the structural conclusion in ref. 12 where, among other things, according to Hartree-Fock 6$31 \mathrm{G}$ calculations for an MC2 homologue bearing a shorter alkyl chain, the energy of the right-side conformer in Fig. 1 is expected to be much higher than that of the left-side one. As also noted in ref. 12, in the dominant left-side conformation, the hydrophilic $\left(-\mathrm{CH}_{2} \mathrm{COOH}\right)$ and hydrophobic $\left(-\mathrm{C}_{18} \mathrm{H}_{36}\right)$ groups point towards opposite directions relative to that of the chromophoric backbone, thus fitting in with the well known ability of MC2 to form stable LB films.

The ${ }^{1} \mathrm{H}$ NMR spectrum of MC1 (chemical shifts are reported in Table SI1, ESI $\dagger$ ), while indicating again a single dominant conformation in solution, does not provide hints useful to identify it between the two in Fig. 1. However, based on the similarity in the LB behaviours of the two merocyanines (see below), the dominant conformation of MC1 may be assumed to be that having a trans arrangement at the central $\mathrm{N}-\mathrm{C}=\mathrm{C}-$ $\mathrm{CO}$ bond sequence, as represented in the left side of Fig. 1.

The absorption spectra of MC1 and MC2 in the near UV and visible regions (Fig. 2) show the colour bands (i.e., the intense bands in the visible spectral region) to be, by far, the most intense ones. These are characterized by a blurred vibronic structure with separations of $c a .900$ and $c a .1000 \mathrm{~cm}^{-1}$, respectively. Moving from cyclohexane to acetonitrile, the colour-band maxima shift from 422 to $426 \mathrm{~nm}\left(-230 \mathrm{~cm}^{-1}\right)$ for MC1 and from 499 to $518 \mathrm{~nm}\left(-740 \mathrm{~cm}^{-1}\right)$ for MC2.

Both compounds exhibit rather low fluorescence quantum yields (Table 2), MC2 being slightly more emissive than MC1

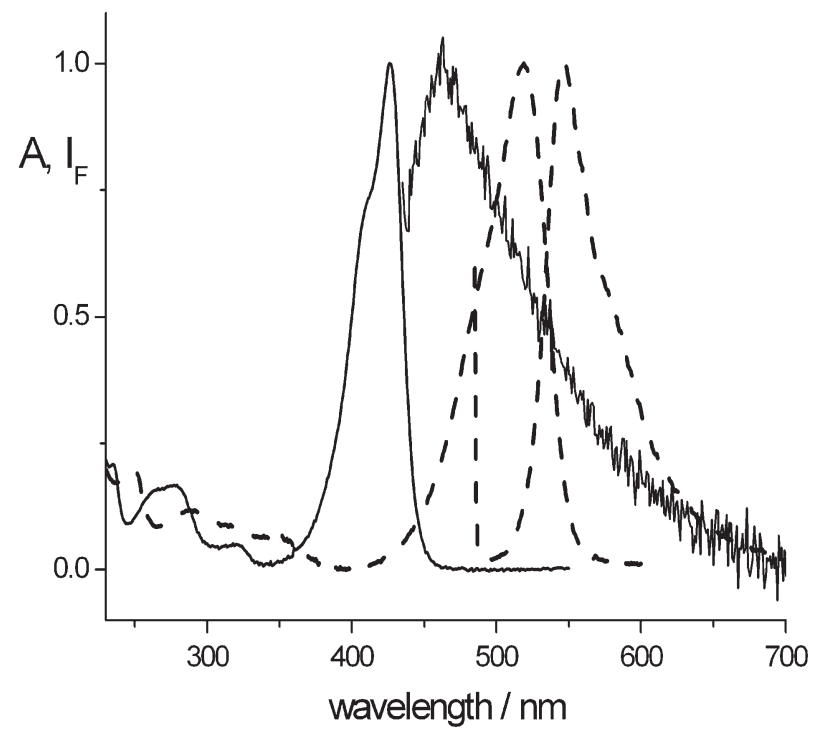

Fig. 2 Absorption and emission spectra of MC1 (solid) and MC2 (dashed) in acetonitrile. The spectra have been normalized at their maxima. Excitation wavelengths: $380 \mathrm{~nm}$ (MC1) and $480 \mathrm{~nm}$ (MC2).

in all solvents. The increase observed in moving from the lowpolarity solvent to the two polar ones suggests that proximity effects, ${ }^{26}$ related to the presence of a low-lying $n \pi *$ state, are active. The slightly lower yields in methanol relative to acetonitrile are consistent with an enhancement of radiationless decays due to solute-solvent hydrogen bonds, with the vibrational modes of the intermolecular hydrogen bond acting as good accepting modes in the radiationless transition. ${ }^{27}$

\subsection{Langmuir monolayers}

The surface-pressure $(\pi)$-area-per-molecule isotherms measured for several $\mathrm{MC}(1 / 2)$ : arachidic acid $\left(\mathrm{CH}_{3}\left(\mathrm{CH}_{2}\right)_{18} \mathrm{COOH}\right.$, AA) binary mixtures are shown in Fig. 3 . With reference to a general, idealized $\pi-A$ diagram, ${ }^{28}$ the isotherms in Fig. 3 describe the two regions corresponding, upon compression, to the expanded liquid state and the transition to the condensed liquid state. ${ }^{29}$ For the condensed phases of pure merocyanines, limit areas per molecule $\left(A_{\pi \rightarrow 0}\right)$ were between 65 and $70 \AA^{2}$ for both dyes, in agreement with published values for MC2 analogues. ${ }^{10}$ As suggested in ref. 10, these limit areas are consistent with the merocyanines lying on water with the long chromophore axis almost parallel to the surface and the short axis rather tilted for MC2, possibly flatter for the shorter, rigid analogue.

Contrary to what happens with MC2, MC1 monolayers did not exhibit a well defined collapse pressure but, rather, subsequent rearrangements with increasing $\pi$. Pure films of

Table 2 Fluorescence quantum yields. $T=20^{\circ} \mathrm{C}$

\begin{tabular}{llll}
\hline & cyclohexane & acetonitrile & methanol \\
\hline MC1 & 0.0004 & 0.001 & 0.0008 \\
MC2 & 0.0007 & 0.003 & 0.0022
\end{tabular}




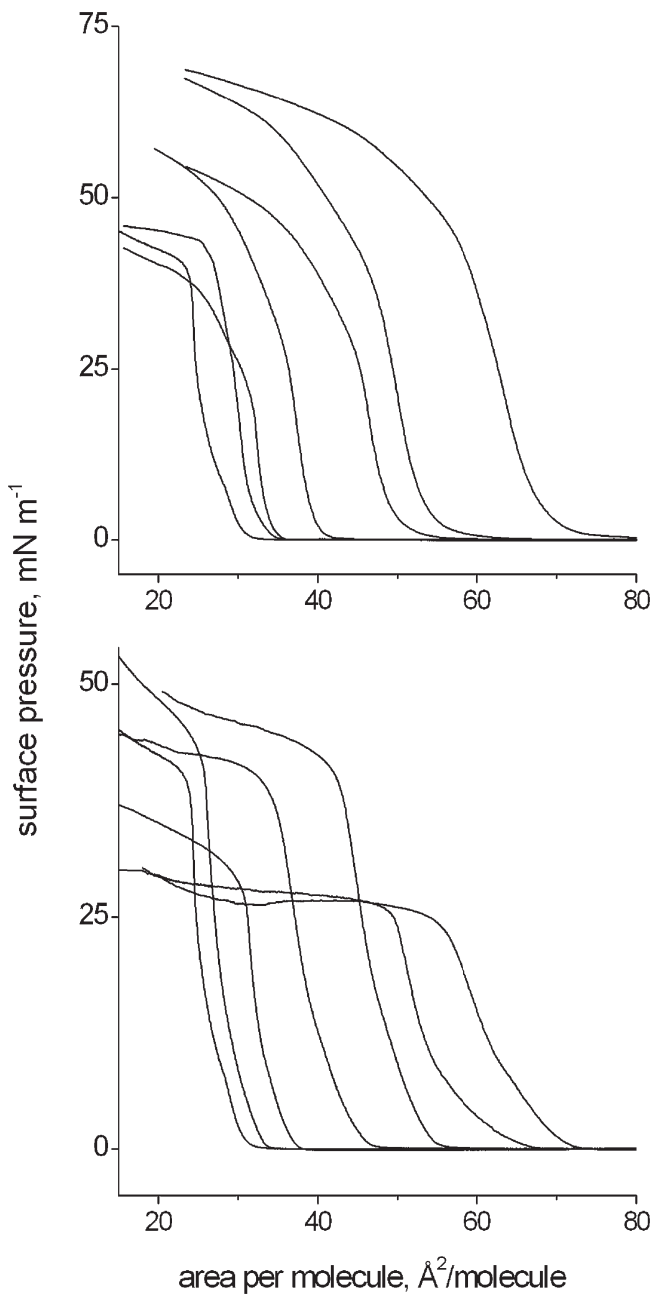

Fig. 3 Surface-pressure $(\pi)$-area-per-molecule isotherms for several merocyanine-arachidic acid binary mixtures. Top: from left to right at $10 \mathrm{mN} \mathrm{M}^{-1}$, $\mathrm{MC1}: \mathrm{AA}=0,0.25,0.5,1,2,4$, pure $\mathrm{MC1}$. Bottom: from left to right at $10 \mathrm{mN}$ $\mathrm{M}^{-1}, \mathrm{MC2}: \mathrm{AA}=0,0.25,0.5,1,2,4$, pure $\mathrm{MC} 2 . T=20^{\circ} \mathrm{C}$.

both merocyanines were fragile, as they sometimes exhibited anticipated collapses. The collapse pressures of the MC2 : AA mixtures were different from those of the pure component films, indicating that the two compounds are not totally immiscible. However, they did not exhibit a regular dependence on mixture composition, thus suggesting that MC2 : AA mixing was not ideal. Compression of $\mathrm{MC}(1 / 2)$ : AA films was not reversible: recompression after expansion was governed by isotherms similar to those of pure AA (Fig. SI1 in ESI†). This suggests that merocyanine aggregates, formed in the first compression, were not disrupted back to floating monomers during the film expansion, and the following compression mainly involved AA molecules.

The dependence on the merocyanine-AA binary mixture composition of the average area per molecule $\left(A_{12}\right)$ and of the relative excess area per molecule $A_{\mathrm{ex}} / A_{\mathrm{id}}$ are shown in Fig. 4 for the two merocyanines at different surface pressures; here $A_{\mathrm{ex}}=$ $A_{12}-A_{\text {id }}=A_{12}-\left(\chi_{1} A_{1}+\chi_{2} A_{2}\right)=A_{12}-A_{2}-\chi_{1}\left(A_{1}-A_{2}\right)$, where $\chi_{1,2}$ are the mole fractions of MC1 and MC2, respectively.

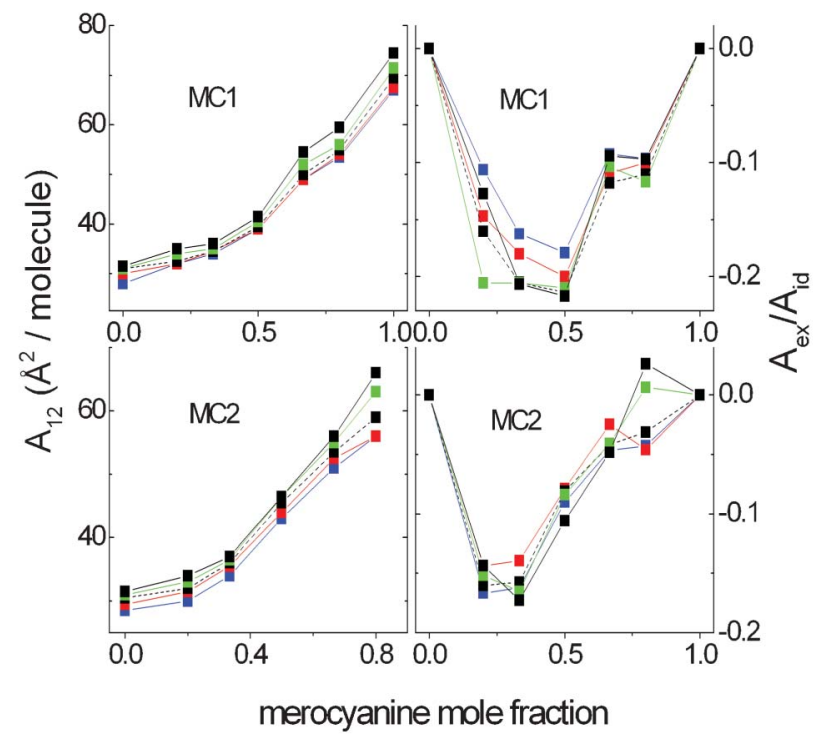

Fig. 4 Dependence of the average areas per molecule $\left(A_{12}\right)$ and of the relative excess areas per molecule $A_{\text {ex }} / A_{\text {id }}$ on merocyanines-AA mixture mole fractions and surface pressure ( $\pi=2$ (black), 5 (green), 10 (black dashed), 15 (red) and 20 (blue) $\mathrm{mN} \mathrm{m}^{-1}$ ).

An ideal behaviour would be characterized by a linear dependence of $A_{12}$ on $\chi$ with $A_{\mathrm{ex}}=0$. Negative, attractive deviations are clearly observed on the left side of Fig. 4 and are displayed as $A_{\mathrm{ex}} / A_{\mathrm{id}}$ on the right side. They are rather pronounced if compared with, e.g., the mixing of octadecylamine and stearic $\operatorname{acid}^{30}$ and of the liposome components, dipalmitoylphosphatidylcholine and dihexadecyl phosphate, on a phosphate buffer subphase. ${ }^{31}$ They are already large at low $\pi$ and increase slowly in the $2-20 \mathrm{mN} \mathrm{m}^{-1}$ range. This indicates that attractive merocyanine-AA forces are operative even in the expanded phase. The effects of intermolecular attraction on molecular packing is largest at an MC1 mole ratio around 0.4 and an $\mathrm{MC} 2$ mole ratio around 0.3 , i.e., roughly, a 1 : 2 merocyanine : AA mole ratio.

Intercomponent interactions, and the corresponding mixed monolayer stability, may be quantified in terms of the excess free energy of mixing, $\Delta G_{\mathrm{ex}}=\int_{0}^{\pi} A_{\mathrm{ex}} \mathrm{d} \pi$ and of the total free energy of mixing, $\Delta G_{\text {mix }}=\Delta G_{\text {id }}+\Delta G_{\text {ex }}$, where $\Delta G_{\text {id }}=R T\left(\chi_{1} \ln \chi_{1}+\right.$ $\left.\chi_{2} \ln \chi_{2}\right){ }^{32}$ Negative $\Delta G_{\mathrm{ex}}$ and $\Delta G_{\text {mix }}$ were obtained from the isotherms of the various binary mixtures (Table 3 ) thus confirming the larger stability of the mixed relative to the corresponding

Table 3 Excess $\left(\Delta G_{\text {ex }}\right)$ and total $\left(\Delta G_{\text {mix }}\right)$ free energies of $M C(1 / 2)$ : AA mixing at the air-water interface. $\boldsymbol{\chi}$ are the mole fractions

$\chi_{\mathrm{MC} 1} \Delta G_{\text {ex }}[\mathrm{J} / \mathrm{mole}] \Delta G_{\mathrm{mix}}[\mathrm{J} / \mathrm{mole}] \chi_{\mathrm{MC} 2} \Delta G_{\mathrm{ex}}[\mathrm{J} / \mathrm{mole}] \Delta G_{\mathrm{mix}}[\mathrm{J} / \mathrm{mole}]$

\begin{tabular}{llllll}
\hline 0.8 & -707.70 & -1918.98 & 0.8 & -150.55 & -1361.83 \\
0.67 & -654.10 & -2194.85 & 0.67 & -228.84 & -1769.59 \\
0.5 & -655.00 & -2332.85 & 0.5 & -449.38 & -2127.23 \\
0.33 & -900.44 & -2441.20 & 0.33 & -718.73 & -2259.49 \\
0.2 & -578.51 & -1789.79 & 0.2 & -446.53 & -1657.81
\end{tabular}


unmixed monolayers. Stabilization is slightly more pronounced for the more rigid dye. Again, the largest stabilization is obtained at a $1: 2$ merocyanine : AA mole ratio. These consistent thermodynamic behaviours point to an enhancement of the dye aggregation induced by both non-specific and specific (hydrogen bonding) dye-AA interactions. ${ }^{11}$

Brewster angle microscopy carried out at the air-water interface indicates that, even at low surface pressures, the surface covering is incomplete and the floating film of pure MC1 appears very rigid (see Fig. SI2 and SI3 in ESI $\dagger$ ). In these conditions, thin and long (30-40 $\mu \mathrm{M})$ mesoscopic structures are visible. By increasing the surface pressure, a complete covering of the surface is obtained, but the film is still not uniform. On the other hand, the pictures taken for a $1: 2$ MC1 : AA mixture (Fig. SI3, ESI $\dagger$ ) indicate a remarkably enhanced homogeneity of the spread film, which do not exhibit the bright, sharp mesoscopic structures observed with pure MC1. Analogous observations were made for MC2 and MC2 : AA films (data not shown). So, with an increasing AA : MC ratio, the floating film uniformity increases and its rigidity decreases, improving the transfer ratio onto the solid support.

\subsection{Langmuir-Blodgett films}

Mono- and multilayers were deposited on hydrophilic quartz slides at surface pressures $\pi=12$ and $20 \mathrm{mN} \mathrm{m}^{-1}$ obtaining very similar transfer ratios, film qualities and spectra. Again, this indicates that rather strong intermolecular interactions also act at the lower deposition surface pressure (i.e., in the expanded phase). With both dyes, $100 \%$ transfer ratios were consistently obtained with $\mathrm{MC}: \mathrm{AA}=1: 2$ and $\mathrm{MC}: \mathrm{AA}=1: 4$ film compositions. With all the other mixtures (see mole compositions in Table 3), 100\% transfer ratios were obtained in the emersion phase, while much less efficient transfers (10$20 \%$ ) were measured in the immersion, leading to a sort of Z-type deposition; also, these films were macroscopically inhomogeneous.

Films deposited with a good transfer ratio (MC : AA $=1: 4$ in the cases shown in Fig. 5) exhibited marked J bands (maxima at 467 and $607 \mathrm{~nm}$ for MC1 and MC2, respectively) traceable to the characteristic head-to-tail arrangement of the chromophores. Z-Type deposited film spectra (4:1 ratio and dashed curves in Fig. 5) featured broader J bands, implying increased disorder in the aggregates, and larger spectroscopic contributions from monomers (M) and $\mathrm{H}$ aggregates (see Fig. 5 and the fluorescence data below for band assignments).

Films obtained with a $\mathrm{MC}: \mathrm{AA}=1: 2$ mole ratio were further characterized. The J band intensity increased linearly with the number of deposited layers (Fig. SI4, ESI $\dagger$ ). All films, but especially monolayers, were however observed to be photolabile: J bands quite rapidly faded away (see ref. 33, and Fig. SI5, ESI $\dagger$ ). So only multilayers were further investigated. LB films obtained from 1:2 MC(1/2) : AA solutions were very homogeneous. When analyzed with atomic force microscopy (surface images and height profiles are shown in Fig. SI6, ESI $\dagger$ ) they exhibited low roughness values. After the very first layers, the roughness increased regularly and very

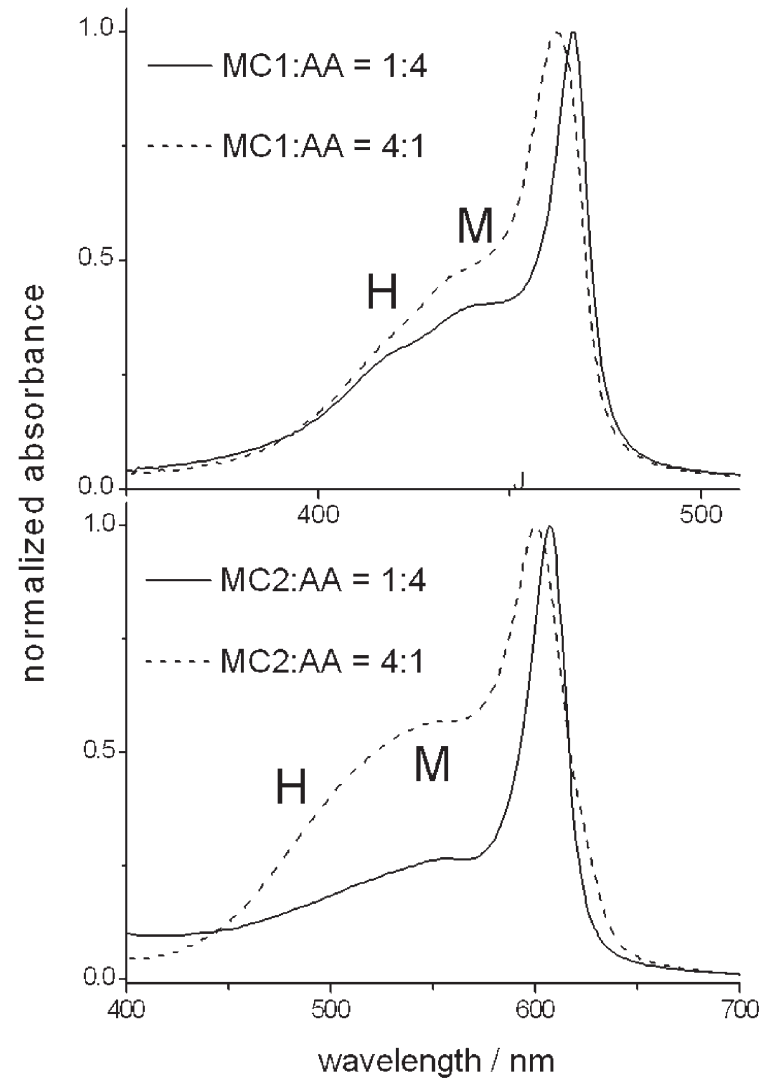

Fig. 5 UV-vis absorption spectra of MC : AA LB films deposited at $20 \mathrm{mN} \mathrm{m}^{-1}$. Samples were made of 9 layers for each quartz substrate side. The spectra were normalized at the maxima for ease of comparison.

slowly, ca. $1 \AA$ A/layer for both dyes, with the number of deposited layers (Table SI2, ESI $\dagger$ ).

The aggregate orientational distributions were investigated by measuring the linear dichroism of LB films made of 29 layers per side of the quartz plate. Measurements were performed at different values of the incidence angle, $\beta=0^{\circ}$ (normal to the film), $30^{\circ}$ and $45^{\circ}$ (these values were corrected for the film refractive index, $\mathrm{n}=1.4,{ }^{34}$ as $\beta_{\text {real }}=\arcsin \left(n^{-1}\right.$ $\sin \beta)) .{ }^{35}$ The $\mathrm{J}$ bands in the films of both dyes exhibited a marked linear dichroism (Fig. 6). Under the simplifying assumption of pure polarization at the J bands, the ensemble-averaged value of the squared cosine of the angle between the $\mathrm{J}$ band transition dipole and the LB-film dipping direction (shown in Fig. 7) holds $\left\langle\cos ^{2} \phi\right\rangle=\frac{D_{0}}{D_{0}+2}$, where $D_{0}$ is the ratio of absorbances measured with vertical (dipping direction) and horizontal polarizations (with $\beta=0^{\circ}$ ). ${ }^{36}$

We find $D_{0}=1.85$ for MC1 and 1.39 for MC2, which yields the $\left\langle\cos ^{2} \phi\right\rangle$ values 0.48 and 0.41 , corresponding to the $\phi$ values $46^{\circ}$ and $50^{\circ}$, respectively. So, the $\mathrm{J}$ aggregates of the two merocyanines in the LB films exhibit a tendency (slightly stronger for MC1) to align with the direction of the $\mathrm{J}$ band dipole transition moment along the substrate dipping direction (named vertical in Fig. 6). 


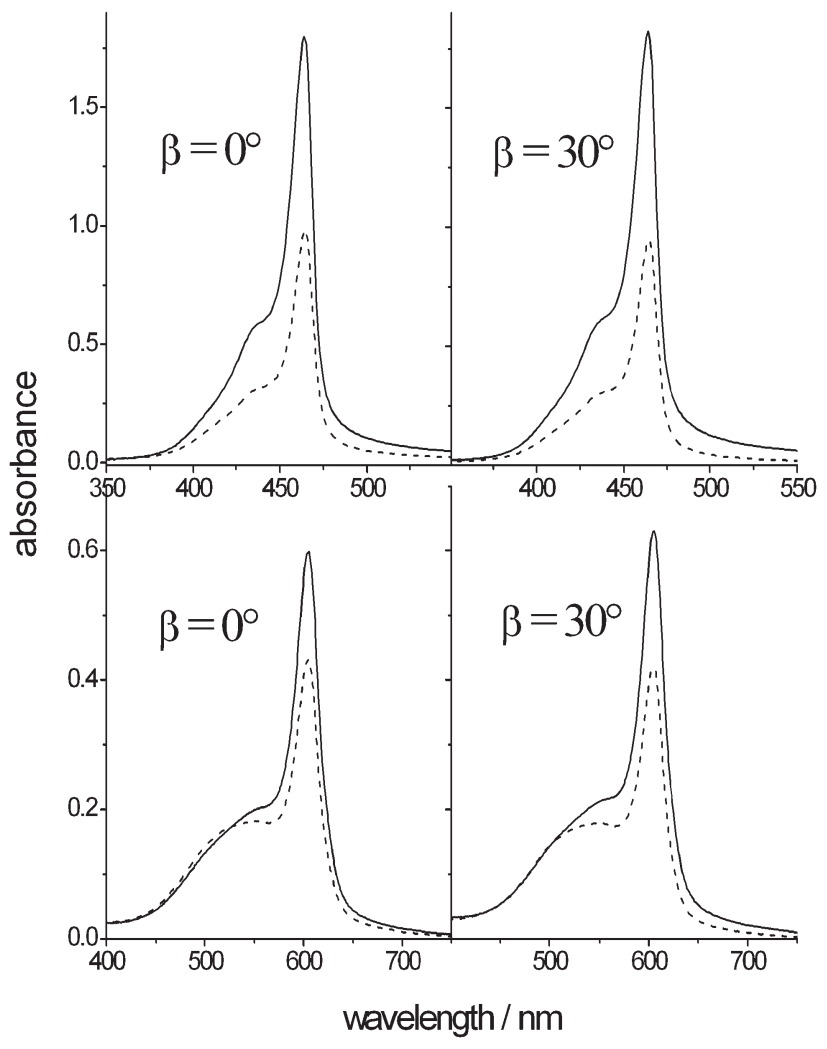

Fig. 6 Absorption spectra of MC1 (top) and MC2 (bottom) LB films measured with vertically (solid lines) and horizontally (dashed lines) polarized light at two incidence angles $(\beta)$. Samples were made of 29 layers per side of the quartz slide.

Measurements at higher incidence angles yielded information on the orientational anisotropy out of the film plane. Application of eqn (1), where $D_{\beta}$ is the dichroic ratio $\left(A_{\text {vert }}\right)$

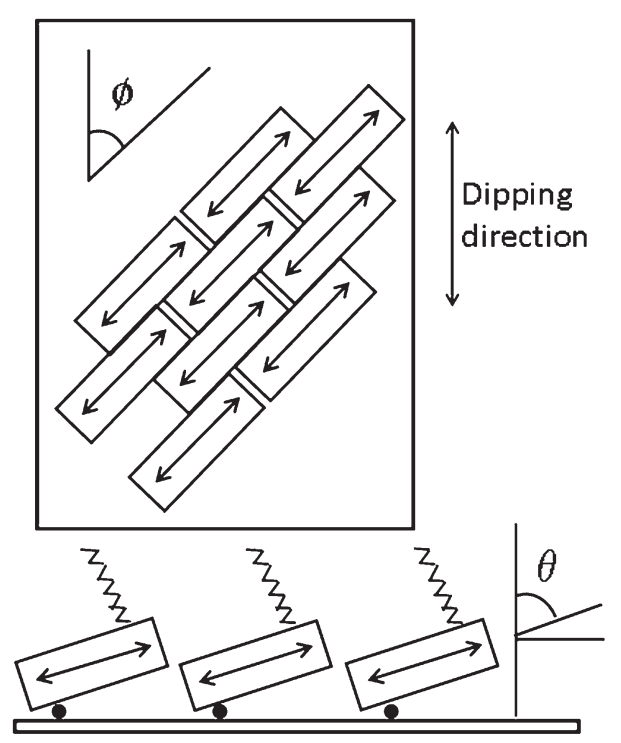

Fig. 7 Schematic representation of a merocyanine J aggregate and of the relevant orientation angles, $\phi$ and $\theta$ (see paragraph 3.3).
$\left.A_{\text {horiz }}\right)$ with the incidence angle $\beta$, enabled us to obtain the average value of $\cos ^{2} \theta, \theta$ being the angle between the $\mathrm{J}$ band dipole transition moment and the normal to the film plane (Fig. 7). ${ }^{35}$

$$
\left\langle\cos ^{2} \theta\right\rangle=\frac{D_{\beta} \cos ^{2} \beta-D_{0}}{\left(1-2 \sin ^{2} \beta\right) D_{\beta}-\left(1+D_{\beta} \sin ^{2} \beta\right) D_{0}}
$$

With the data for the J bands in Fig. 6, we obtained $\left\langle\cos ^{2} \theta\right\rangle$ $=0.17$ and 0.12 , roughly corresponding to $\theta \approx 65^{\circ}$ and $70^{\circ}$, for MC1 and MC2, respectively. Therefore, both dyes tend to lie with the direction of the $\mathrm{J}$ band polarization only slightly tilted $\left(20-25^{\circ}\right)$ relative to the film surface. $\mathrm{H}$ aggregate absorption was well observable only for MC2 as a broad shoulder below $520 \mathrm{~nm}$. Within this band both $D_{0}$ and $D_{30}$ were around 1 , which indicates a completely isotropic orientation distribution of the $\mathrm{H}$ aggregates $\left(\phi \sim \theta \sim 55^{\circ}\right)$. For MC1, in the $\lambda<420 \mathrm{~nm}$ tail, the presumed region of $\mathrm{H}$-aggregate absorption, we calculate $D_{0} \sim 1.6$, corresponding to a $\phi$ value $\mathrm{ca} .48^{\circ}$ and $D_{30} \sim 1.76$, corresponding to a $\theta$ value around $70^{\circ}$. These values are very similar to those of the $\mathrm{J}$ aggregates, probably because of the very low absorption contributions from the $\mathrm{H}$ aggregates found with the shorter merocyanine.

Some fluorescence spectra of $1: 2 \mathrm{MC}(1 / 2)$ : AA 9-layer films are reported in Fig. 8. Both emissions are dominated by $\mathrm{J}$ bands with maxima at 485 and $633 \mathrm{~nm}$, respectively. Weaker and broader H-type bands are observed around 520 and 675 $\mathrm{nm}$. These assignments were confirmed by the correspondence of the excitation bands with the absorption bands. While contributions from monomers were discernible in the absorption spectra of Fig. 5, 6 and 8 (around 440 and $540 \mathrm{~nm}$, respectively), no significant emission with excitation corresponding to these regions was found; this is likely due to the low fluorescence quantum yields of the monomeric merocyanines (Table 2).

Films deposited at a high surface pressure, $\pi=25 \mathrm{mN} \mathrm{m}^{-1}$, exhibited a different spectroscopic behaviour for the two dyes. While the spectra of MC1 were the same as seen at lower surface pressures, MC2 showed a doubling of the J band (panel c in Fig. 8), which indicates the presence of two different $\mathrm{J}$ aggregates. In addition to the previously observed $607 \mathrm{~nm}$ band, a new absorption shoulder, which becomes a narrow band in the excitation spectrum, was observed around $625 \mathrm{~nm}$. This occurred at all MC2 : AA ratios, though not with a reproducible relative intensity. The additional J aggregate was already present, though in smaller amounts, in the MC2 films deposited at $20 \mathrm{mN} \mathrm{m}^{-1}$ (see panel b in Fig. 8). In all cases, a single emission was observed with a maximum at $633 \mathrm{~nm}$. This implies that, in these films, migration of excitons of both kinds occurred to a single J-type emitting trap. Linear dichroism spectra (Fig. SI7, ESI $\dagger$ ) show that both J aggregate types align with the components of the transition dipole moment on the film plane parallel to the dipping direction. On the other hand, we find $\theta=57^{\circ}$ for the $\mathrm{J}_{\mathrm{B}}$ red-shifted band, corresponding to an almost isotropic distribution of orientations of the $\mathrm{J}$ transition moment out of the film plane; for the $\mathrm{J}_{\mathrm{A}}$ band we obtain $\theta=61^{\circ}$ rather than $\theta=70^{\circ}$, as found for films deposited at $20 \mathrm{mN} \mathrm{m}^{-1}$, possibly as a consequence of 


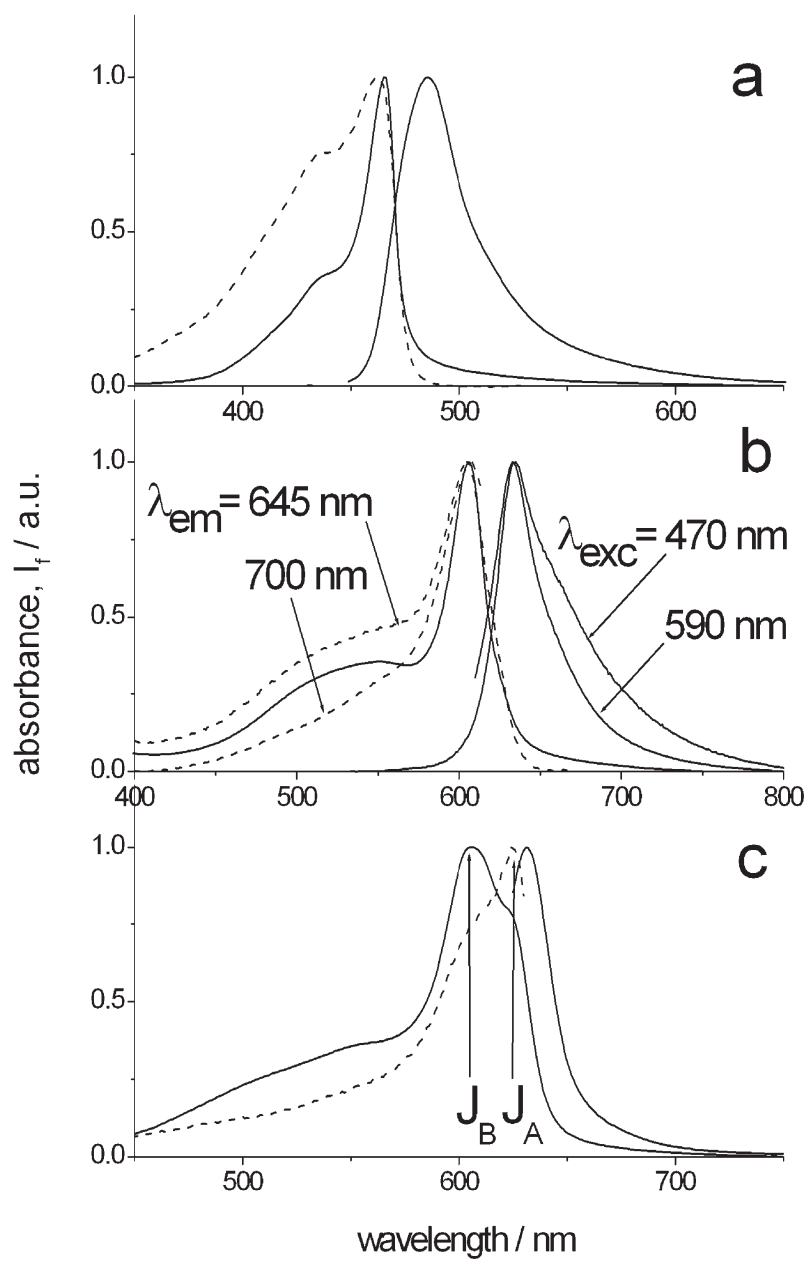

Fig. 8 Absorption (solid) and fluorescence emission and excitation (dashed) spectra of 9-layer films: (a) $1: 2 \mathrm{MC1}: \mathrm{AA}, \pi=20 \mathrm{mN} \mathrm{m}^{-1}, \lambda_{\mathrm{exc}}=430 \mathrm{~nm}, \lambda_{\mathrm{em}}=$ $540 \mathrm{~nm}$; (b) $1: 2 \mathrm{MC2}: \mathrm{AA}, \pi=20 \mathrm{mN} \mathrm{m}^{-1}$; (c) $1: 2 \mathrm{MC2}: \mathrm{AA}, \pi=25 \mathrm{mN} \mathrm{m}^{-1}$, $\lambda_{\text {exc }}=610 \mathrm{~nm}, \lambda_{\text {em }}=660 \mathrm{~nm}$. All spectra have been normalized at the maxima for ease of presentation.

the evident spectral overlap of the two J bands. There is therefore some evidence of a more markedly in-plane alignment of the hypsochromically-absorbing J aggregates, suggesting that the bathochromically-absorbing ones might result from protrusions out of the air-water surface occurring before deposition at the higher surface pressure.

\section{Conclusions}

The amphiphilic MC2 dye, having pentamethine-merocyanine as the basic chromophore, has been long known to form stable $\mathrm{J}$ aggregates in LB films which can be of prime interest for applications in photonic devices. In the present paper we have extended the study to an homologous dye, MC1, based on the shorter trimethine-merocyanine chromophore, aiming to find out the effects of the chromophore length, other structural features being almost unchanged, on the properties of the LB films prepared under the same conditions. For this purpose, we have carried out a strictly parallel analysis for the two dyes, MC1 and MC2. In order to emphasize the role of the different molecular components, our study encompassed three levels of increasing complexity: (1) the structural and spectroscopic properties of the monomers in solution; (2) the thermodynamic and morphological properties of the Langmuir monolayers at the air-water interface; (3) the spectroscopic and photophysical characterization of the aggregates in the LB films. The main point-by-point conclusions follow.

A new ${ }^{1} \mathrm{H}$ NMR study confirmed previous conclusions drawn for MC2 on the basis of different spectroscopic observables and showed that, in chloroform solutions, the two dyes adopt conformations with an s-trans arrangement of the respective polymethine chains where the hydrophilic and hydrophobic groups point to mutually opposite directions: this is likely a pre-condition for an ordered molecular arrangement to be obtained on an aqueous subphase.

In the Langmuir monolayers doped with arachidic acid both dyes are subjected to attractive interactions with the molecules of the fatty acid. The stability of the monolayer has a maximum for the $1: 2 \mathrm{MC}(1 / 2)$ : AA mixture. On average, the stability of the MC1 monolayers is higher than that of the MC2 monolayers, as evidenced by more negative values of $\Delta G_{\text {mix }}$. From the analysis of the limiting areas, $A_{\pi \rightarrow 0}$, the tilting angle of the MC1 chromophore with respect to the subphase surface appears to be slightly smaller than that adopted by the MC2 chromophore, a difference possibly traceable to the different lengths of the alkyl chains.

LB multilayers can be Y-deposited at $12 \mathrm{mN} \mathrm{m}^{-1}<\pi<25$ $\mathrm{mN} \mathrm{m}{ }^{-1}$ with good transfer ratios only for $\mathrm{MC}: \mathrm{AA}=1: 4$ and $\mathrm{MC}: \mathrm{AA}=1: 2$. At higher $\mathrm{MC}$ mole ratios, interlayer repulsion caused very inefficient deposition in the immersion phase, and these Z-deposited multilayers were macroscopically inhomogeneous.

The spectroscopically and photophysically dominant species in the LB multilayers are $\mathrm{J}$ aggregates for both merocyanines, although some large $\mathrm{H}$ aggregates contribute to the MC2 film spectra. From their absorption linear dichroism, we conclude that MC1 and MC2 J aggregates preferentially align with their transition dipoles along the dipping direction and slightly tilted relative to the substrate surface. Both these tendencies are slightly more pronounced for the J aggregates of MC1. In this respect, a significant difference between the two dyes in the LB films is a J aggregate dimorphism observed for MC2, but not for MC1, in the same conditions. The two J absorption/excitation bands observed for MC2, particularly at high deposition $\pi$ values, indicate the coexistence of two types of J aggregates, which are characterized by different linear dichroisms and, therefore, average alignments. Exciton migration occurs efficiently from the higher to the lower excitation-energy J sites. These observations are consistent with the occurrence of different $\mathrm{J}$ aggregates in protrusions of MC2 films out of the substrate surface. In this respect too, MC1 layers appear slightly more solid and ordered, though this difference is not observable in AFM surface images. 
Because of its excellent J-aggregating properties in LB films, MC1 represents a new promising chromophore for photonic applications, particularly the non-linear ones that take advantage of the extensive formation and ordered arrangement of J aggregates. ${ }^{37}$ Additionally, the 140-150 nm hypsochromic shifts of the main linear absorption band and of the emission band of the MC1 J aggregates, with respect to those of MC2, significantly broaden the working wavelength range for such applications.

\section{Acknowledgements}

Dr M. Cecilia Rossi of CIGS (University of Modena and Reggio Emilia) is thanked for carrying out the NMR experiments.

\section{References}

1 O. Wörz and G. Scheibe, Z. Naturforsch., 1969, 146, 381.

2 L. Franco, L. Pasimeni, G. Ponterini, M. Ruzzi and U. Segre, Phys. Chem. Chem. Phys., 2001, 3, 1736.

3 S. Kuroda, Adv. Colloid Interface Sci., 2004, 111, 181.

4 Y. Hirano, S. Tateno, A. Maio and Y. Ozaki, J. Phys. Chem. B, 2009, 113, 2764.

5 K. J. Saito, J. Phys. Chem. B, 1999, 103, 6579.

6 K. Saito and S. Kobayashi, Appl. Phys. Lett., 2002, 80, 1489.

7 K. Kato, M. Terakado, K. Shinbo, F. Kaneko and T. Wakamatsu, Thin Solid Films, 2001, 393, 97.

8 Y. Hamanaka, H. Kurosawa, A. Nakamura, Y. Uchiyama, K. Marumoto and S. Kuroda, Chem. Phys. Lett., 2002, 363, 233.

9 F. Momicchioli, G. Ponterini and D. Vanossi, J. Phys. Chem. A, 2008, 112, 11861.

10 H. Nakahara, K. Fukuda, D. Möbius and H. Kuhn, J. Phys. Chem., 1986, 90, 6144.

11 K. Ikegami, C. Mingotaud and M. Lan, J. Phys. Chem. B, 1999, 103, 11261.

12 K. Ikegami and S. Kuroda, Chem. Phys., 2003, 295, 205.

13 M. Murata, K. Mori, A. Sakamoto, M. Villeneuve and H. Nakahara, Chem. Phys. Lett., 2005, 405, 32.
14 Y. Hirano, A. Maio and Y. Ozaki, Langmuir, 2008, 24, 3317. 15 Y. Hirano, S. Tateno and Y. Ozaki, Langmuir, 2007, 23, 7003.

16 N. Kato, K. Saito and Y. Uesu, Thin Solid Films, 1999, 338, 5.

17 A. Miyata, D. Heard, Y. Unuma and Y. Higashigaki, Bull. Chem. Soc. Jpn., 1993, 66, 999.

18 H. Nakahara and D. Möbius, J. Colloid Interface Sci., 1986, 114, 363.

19 A. Lodi, M. Caselli, A. Casnati, F. Momicchioli, F. Sansone, D. Vanossi and G. Ponterini, J. Mol. Struct., 2007, 846, 49.

20 N. Kato, K. Saito, H. Aida and Y. Uesu, Chem. Phys. Lett., 1999, 312, 115.

21 T. Schneider and O. D. Lavrentovich, Langmuir, 2000, 16, 5227.

22 S. L. Murov, I. Carmichael and G. L. Hug, Handbook of Photochemistry, 2nd edn, Dekker, New York, 1993, ISBN 0-8247-7911-8.

23 P. G. Seybold, M. Gouterman and J. Callis, Photochem. Photobiol., 1969, 9, 229.

24 T. Kawaguchi and K. Iwata, Thin Solid Films, 1988, 165, 323.

25 H. Funakoshi, K. Tamagache and Y. Fujita, Nippon Kagaku Kaishi, 1989, 56-62.

26 E. C. Lim, J. Phys. Chem., 1986, 90, 6770.

27 H. Inoue, M. Hida, N. Nakashima and K. Yoshihara, J. Phys. Chem., 1982, 86, 3184.

28 M. S. Aston, Chem. Soc. Rev., 1993, 22, 67.

29 E. Kiss, A. Vargha and E. I. Vargha-Butler, Phys. Chem. Chem. Phys., 2004, 6, 1575.

30 Y.-L. Lee, Y.-C. Yang and Y.-J. Shen, J. Phys. Chem. B, 2005, 109, 4662.

31 T.-H. Chou and C.-H. Chang, Langmuir, 2000, 16, 3385.

32 P. Dynarowicz-Łatka and K. Kita, Adv. Colloid Interface Sci., 1999, 79, 1.

33 H. C. Yoon, J. H. Son, J. M. Kim and Y. S. Kwon, Opt. Mater., 2002, 21, 407.

34 L. Pasimeni, M. Meneghetti, R. Rella, L. Valli, C. Granito and L. Troisi, Thin Solid Films, 1995, 265, 58.

35 A. Lodi and G. Ponterini, Thin Solid Films, 2006, 496, 585.

$36 \mathrm{~J}$. Michl and E. W. Thulstrup, Spectroscopy with polarized light, VCH Publishers, New York, 1986, ch. 5.

37 E. Collini, C. Ferrante, R. Bozio, A. Lodi and G. Ponterini, J. Mater. Chem., 2006, 16, 1573. 